\title{
Effect of welding variables on mechanical properties of low carbon steel welded joint
}

\author{
Talabi, S.I. ${ }^{a}{ }^{,},{ }^{\prime}$ Owolabi, O.B. ${ }^{b}$, Adebisi, J.A. ${ }^{a}$, Yahaya, T. ${ }^{a}$ \\ ${ }^{a}$ Department of Materials and Metallurgical Engineering, University of Ilorin, Ilorin, Nigeria \\ ${ }^{\mathrm{b}}$ National Engineering Design Development Institute, Nnewi Anambra State, Nigeria
}

\section{A B S T R A C T}

This paper discussed the effect of welding variables on the mechanical properties of welded $10 \mathrm{~mm}$ thick low carbon steel plate, welded using the Shielded Metal Arc Welding (SMAW) method. Welding current, arc voltage, welding speed and electrode diameter were the investigated welding parameters. The welded samples were cut and machined to standard configurations for tensile, impact toughness, and hardness tests. The results showed that the selected welding parameters had significant effects on the mechanical properties of the welded samples. Increases in the arc voltage and welding current resulted in increased hardness and decrease in yield strength, tensile strength and impact toughness. Increasing the welding speed from $40-66.67 \mathrm{~mm} / \mathrm{min}$ caused an increase in the hardness characteristic of the welded samples. Initial decrease in tensile and yield strengths were observed which thereafter increased as the welding speed increased. An electrode diameter of $2.5 \mathrm{~mm}$ provided the best combination of mechanical properties when compared to the as received samples. This behaviour was attributed to the fact that increased current and voltage meant increased heat input which could create room for defect formation, thus the observed reduced mechanical properties.
\end{abstract}

\section{ARTICLE INFO}

Keywords:

Welding

Low carbon steel

Welding variables

Mechanical properties

*Corresponding author: isaacton@yahoo.com

(Talabi, S.I.)

Article history:

Received 18 September 2014

Revised 3 November 2014

Accepted 10 November 2014 


\section{References}

[1] Armentani, E., Esposito, R., Sepe, R. (2007). The effect of thermal properties and weld efficiency on residual stresses in welding, Journal of Achievements in Materials and Manufacturing Engineering, Vol. 20, No. 1-2, 319-322.

[2] Jariyaboon, M., Davenport, A.J., Ambat, R., Connolly, B.J., Williams, S.W., Price, D.A. (2007). The effect of welding parameters on the corrosion behaviour of friction stir welded AA2024-T351, Corrosion Science, Vol. 49, No. 2, 877-909, doi: 10.1016/j.corsci.2006.05.038.

[3] Karadeniz, E., Ozsarac, U., Yildiz, C. (2007). The effect of process parameters on penetration in gas metal arc welding processes, Materials \& Design, Vol. 28, No. 2, 649-656, doi: 10.1016/j.matdes.2005.07.014.

[4] Lothongkum, G., Viyanit, E., Bhandhubanyong, P. (2001). Study on the effects of pulsed TIG welding parameters on delta-ferrite content, shape factor and bead quality in orbital welding of AISI 316L stainless steel plate, Journal of Materials Processing Technology, Vol. 110, No. 2, 233-238, doi: 10.1016/S0924-0136(00)00875-X.

[5] Lothongkum, G., Chaumbai, P., Bhandhubanyong, P. (1999). TIG pulse welding of 304L austenitic stainless steel in flat, vertical and overhead positions, Journal of Materials Processing Technology, Vol. 89-90, 410-414, doi: 10.1016/S0924-0136(99)00046-1.

[6] Mirzaei, M., Arabi Jeshvaghani, R., Yazdipour, A., Zangeneh-Madar, K. (2013). Study of welding velocity and pulse frequency on microstructure and mechanical properties of pulsed gas metal arc welded high strength low alloy steel, Materials \& Design, Vol. 51, 709-713, doi: 10.1016/j.matdes.2013.04.077.

[7] Sakthivel, T., Sengar, G.S., Mukhopadhyay, J. (2009). Effect of welding speed on microstructure and mechanical properties of friction-stir-welded aluminum, The International Journal of Advanced Manufacturing Technology, Vol. 43, No. 5-6, 468-473, doi: 10.1007/s00170-008-1727-7.

[8] Razal Rose, A., Manisekar, K., Balasubramanian, V. (2012). Influences of welding speed on tensile properties of friction stir welded AZ61A magnesium alloy, Journal of Materials Engineering and Performance, Vol. 21, No. 2, 257-265, doi: 10.1007/s11665-011-9889-0.

[9] Afolabi, A.S. (2008). Effect of electric arc welding parameters on corrosion behaviour of austenitic stainless steel in chloride medium, AU Journal of Technology, Vol. 11, No. 3, 171-180.

[10] Lee, J.I., Um, K.W. (2000). A prediction of welding process parameters by prediction of back-bead geometry, Journal of Materials Processing Technology, Vol. 108, No. 1, 106-113, doi: 10.1016/S0924-0136(00)00736-6.

[11] Agarwal, R.L. (1992). Welding engineering - a textbook for engineering students, $4^{\text {th }}$ edition, New Delhi, India, Khanaa Publishers.

[12] Sahin, M. (2005). Joining with friction welding of high-speed steel and medium-carbon steel, Journal of Materials Processing Technology, Vol. 168, No. 2, 202-210, doi: 10.1016/i.jmatprotec.2004.11.015.

[13] Das, C.R., Albert, S.K., Bhaduri, A.K., Srinivasan, G., Murty, B.S. (2008). Effect of prior microstructure on microstructure and mechanical properties of modified $9 \mathrm{Cr}-1 \mathrm{Mo}$ steel weld joints, Materials Science and Engineering: A, Vol. 477, No. 1-2, 185-192, doi: 10.1016/i.msea.2007.05.017.

[14] Boumerzoug, Z., Raouache, E., Delaunois, F. (2011). Thermal cycle simulation of welding process in low carbon steel, Materials Science and Engineering: A, Vol. 530, 191-195, doi: 10.1016/j.msea.2011.09.073.

[15] Gharibshahiyan, E., Raouf, A.H., Parvin, N., Rahimian, M. (2011). The effect of microstructure on hardness and toughness of low carbon welded steel using inert gas welding, Materials \& Design, Vol. 32, No. 4, 2042-2048, doi: $\underline{10.1016 / \text { j.matdes.2010.11.056. }}$ 


\title{
Vpliv spremenljiv pri varjenju na mehanske lastnosti zvarnih spojev na nizkoogljičnem jeklu
}

\author{
Talabi, S.I. ${ }^{\mathrm{a},{ }^{*}}$, Owolabi, O.B. ${ }^{\mathrm{b}}$, Adebisi, J.A. $^{\mathrm{a}}$, Yahaya, $^{\mathrm{T}} .^{\mathrm{a}}$ \\ ${ }^{a}$ Department of Materials and Metallurgical Engineering, University of Ilorin, Ilorin, Nigeria \\ ${ }^{b}$ National Engineering Design Development Institute, Nnewi Anambra State, Nigeria
}

\begin{abstract}
POVZETEK
Članek proučuje vpliv procesnih spremenljivk pri varjenju na mehanske lastnosti $10 \mathrm{~mm}$ debele varjene plošče, narejene iz nizkoogljičnega jekla. Uporabili smo metodo obločnega varjenja z oplaščeno elektrodo (angl. Shielded Metal Arc Welding - SMAW). Procesne spremenljivke so bile varilni tok in napetost, hitrost varjenja in premer elektrode. Pripravili smo standardne preizkušance za natezni preizkus, preizkus udarne žilavosti in merjenje trdote. Rezultati merjenj so pokazali, da imajo izbrane spremenljivke pri varjenju bistven vpliv na mehanske lastnosti varjenih vzorcev. Višja varilna napetost in večji varilni tok omogočata višjo trdoto, medtem ko so se vrednosti za mejo plastičnosti, natezno trdnost in udarno žilavost znižale. Pri povečanju hitrosti varjenja od $40 \mathrm{~mm} / \mathrm{min}$ do $66.67 \mathrm{~mm} / \mathrm{min}$ beležimo povečanje trdote varjenih vzorcev. Začetnemu znižanju vrednosti za natezno trdnost in mejo plastičnosti je sledilo povečanje vrednosti, ko smo povečali hitrost varjenja. Najboljše mehanske lastnosti smo ugotovili na vzorcih, ki so bili varjeni s premerom elektrod 2.5 $\mathrm{mm}$. To pripisujemo dejstvu, da večje vrednosti tokov in višje napetosti pri varjenju pomenijo tudi večje vnose energije, kar lahko povzroči oblikovanje defektov v materialu, ti pa znižujejo mehanske lastnosti zvarov.
\end{abstract}

\section{PODATKI O ČLANKU}

Ključne besede:

Varjenje

Nizkoogljično jeklo

Spremenljivke pri varjenju

Mehanske lastnosti

*Kontaktna oseba:

isaacton@yahoo.com

(Talabi, S.I.)

Zgodovina članka:

Prejet 18. septembra 2014

Popravljen 3. novembra 2014

Sprejet 10. novembra 2014 